\title{
Los aportes de Liliana Sinisi, recuperando debates en torno a los procesos de integración-inclusión / exclusión escolar
}

\author{
Macarena Romero Acuña* y Cristina Pereyra**
}

\section{Resumen}

En este artículo nos proponemos presentar, a modo de homenaje a la antropóloga y profesora Liliana Sinisi, algunos de sus valiosos aportes, que dejaron huellas significativas en la lectura y análisis de nuestros trabajos de campo en torno a los procesos de integración-inclusión educativa. Consideramos que Liliana Sinisi representa un referente muy importante no sólo entre las y los compañeras y compañeros con quienes realizó distintas investigaciones, sino también entre investigadores/as y estudiantes de distintas universidades nacionales y latinoamericanas y entre maestros/as de diferentes escuelas de nuestro país, lo que demuestra su reconocida trayectoria y su paso por ámbitos educativos muy diversos. Partiremos de recuperar la trayectoria de Liliana Sinisi y sus prácticas formativas. Luego, retomando los distintos intercambios realizados con nosotras y su producción académica, nos interesa entrecruzar trabajos de campo realizados en la ciudad de Rosario, Santa Fe, y en la ciudad de Sarmiento, Chubut, entre los años 2008 y 2013. Sobre estas bases, buscamos dar cuenta de los aportes significativos y los procesos de análisis construidos en el desarrollo de nuestras investigaciones. Nos centraremos en aquellos conceptos claves y categorías teóricas y sociales que fuimos poniendo en juego y que construimos a partir de los aportes que Liliana nos fue sugiriendo en relación con la problemática de los denominados procesos de integración-inclusión/exclusión escolar.

*Licenciada en Antropología. Becaria CONICET/CEACU-UNR. Doctoranda en Doctorado en Estudios Sociales de América Latina. Centro de Estudios Avanzados. Universidad Nacional de Córdoba. Rosario, Argentina. Correo electrónico: macarenaromeroa@gmail.com.ar

**Profesora y Licenciada en Ciencias de la Educación. Facultad de Humanidades y Ciencias Sociales. Universidad Nacional de la Patagonia San Juan Bosco. Conicet. Comodoro Rivadavia. Argentina. Correo electrónico: pereyra.cristina@gmail.com

\section{Palabras Clave}

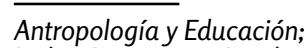
Inclusión-integración educativa; Exclusión;

Educación especial;

Prácticas docentes 


\section{Liliana Sinisi's contributions to the debates on school integration- inclusion/exclusion processes}

\begin{abstract}
Key words

Anthropology and Education; Educational inclusion-

integration;

Exclusion;

Special education; Teaching practices

As a tribute to anthropologist and teacher Liliana Sinisi, this article examines her valuable contributions to the discussion on processes of educational integrationinclusion, which has left significant marks on our fieldwork and research on the topic. We consider Liliana Sinisi a very important reference not only among her peers and colleagues, but also among researchers and students from different national and Latin American universities and among teachers from different schools in our country, thus demonstrating her renowned trajectory and contribution to diverse educational fields. We start by reconstructing Liliana Sinisi's educational and training trajectory. Next, drawing from our exchange with her and from discussions with her academic production, we cross-analyze fieldwork carried out in the city of Rosario, Santa Fe and in the city of Sarmiento, Chubut between 2008 and 2013. On this basis, we seek to account for the significant contributions and analysis processes built over the development of our research. We focus on key concepts and theoretical and social categories that we constructed with suggested contributions from Liliana in relation to the problem of the so-called processes of school integration-inclusion/exclusion.
\end{abstract}

\section{As contribuições de Liliana Sinisi, recuperando debates ao redor dos processos de integração inclusão/exclusão escolar}

\section{Resumo}

Palavras chave

Antropología e Educação; Inclusão-integração educacional; Exclusão; Educação especial; Práticas de ensino
Neste artigo apresentamos, a maneira de homenagem á antropóloga e professora Liliana Sinisi, alguns dos seus valiosos aportes que deixaram marcas significativas nas leituras e análises dos nossos trabalhos de campo en torno aos processos de integração-inclusão educativa. Consideramos que Liliana Sinisi representa um referente muito importante não somente entre suas e seus colegas com os quais fez diferentes pesquisas, senão também entre os e as pesquisadores/as e estudantes de diferentes universidades nacionais e Latino-americanas assim como entre docentes de diferentes escolas do nosso país, demostrando assim sua reconhecida trajetória e seu passo por âmbitos educativos muito diversos. Começaremos por recuperar o percurso de Liliana Sinisi e suas prácticas formativas. Depois, retomando os distintos intercâmbios realizados com nos e sua produção académica interessa-nos conectar trabalhos de campo feitos nas cidades de Rosario, província de Santa Fé e Sarmiento, província de Chubut, entre os anos 2008 e 2013. Sobre estas bases, procuramos dar conta dos significativos aportes e os processos de análise construídos no desenvolvimento das nossas pesquisas. Centraremos-nos em aqueles conceitos chave e as categorias teóricas e sociais que fomos utilizando e que construímos a partir dos aportes que Liliana nos sugeriu com relação à problemática dos denominados processos de integração-inclusão/exclusão escolar. 


\section{Introducción ${ }^{1}$}

En la introducción nos gustaría presentar a Liliana Sinisi, a quien queremos homenajear por su reconocida trayectoria y sus valiosos aportes para el campo de la antropología y la educación. Su recorrido profesional fue muy extenso y diverso, lo que demuestra la riqueza en su formación como investigadora y docente. Formación que a su vez, siempre socializó. Para la reconstrucción de su trayectoria, partimos del análisis de su currículum vitae, junto con la recuperación de recuerdos de algunas de sus compañeras de trabajo a través de distintos diálogos y comunicaciones vía correo electrónico que mantuvimos con ellas durante la elaboración de este escrito. Luego, vincularemos esto con nuestras experiencias formativas junto a ella, para finalmente pensar sus aportes a nuestras problemáticas de investigación, realizadas entre 2008 y 2013.

\section{Liliana...}

"Lili es de esa generación que no responde al estereotipo actual de pura vida académica... La dictadura, lograr la independencia económica, armar familia, pasión por vivir y abrirse a nuevos desafíos hizo que su trayectoria se ampliara, se diversificara y no fuera lineal...." (P.M., 18/02/2018).

En el año 1992 se recibió de Licenciada en Ciencias Antropológicas por la Facultad de Filosofía y Letras de la Universidad de Buenos Aires (UBA). Desde el año 1999, quedó a cargo de la materia Didáctica especial y Prácticas de la Enseñanza (Departamento de Ciencias Antropológicas), como jefa de Trabajo Prácticos, asignatura de la cual fue fundadora. Hasta ese momento, en la carrera de Antropología no existía una didáctica de la antropología, con lo cual, Liliana Sinisi sentó las bases de una propuesta que dio lugar a la formación de los/as antropólogos/a para el trabajo de la enseñanza. Durante el tiempo que permaneció en este espacio formativo construyó un equipo de cátedra que fue creciendo y formando a aquellos profesores y profesoras que aún hoy la conforman.

Nos recuerda L.C. que Liliana ejerció como profesora entre los años 1993 y 2000 en la materia Antropología en el Ciclo Básico Común, junto a la profesora Mirta Lischetti, y en la asignatura Antropología Sistemática I entre los años 1996 y 1997, con la profesora María Rosa Neufeld.

En el posgrado fue profesora de las maestrías en Antropología Social, Psicología Educativa y Aerotécnicos de la UBA. Asimismo, periódicamente dictaba seminarios de grado de Antropología y Educación, y era capacitadora docente en el nivel terciario del Ministerio de Educación de Córdoba.

Comenta E.C. que Liliana trabajaba en Córdoba con los institutos de formación docente. Llegó allí a través de Roxana Mercado (del equipo de M. Maldonado). "Lili hizo allí toda una experiencia de formación en antropología para los formadores" (E.C., 17/02/2018).

Es de resaltar en su trayectoria que desde principios/mediados de los noventa empezó a formar parte como investigadora del Programa de Antropología y Educación, en los equipos dirigidos por María Rosa Neufeld.

en 1992 ya que M. Rosa nos invitó a integrar el Programa de Antropología y Educación que se estaba reacomodando... y también se suma S. A partir de ahí compartimos los UBACYT, la formación inicial como investigadoras y sobre todo un trayecto de intensa amistad y mucha producción conjunta... (P.M. 18/o2/2018).

\author{
1. Este artículo se desprende de \\ una ponencia presentada en las \\ Jornadas Rosarinas de Antropolo- \\ gía Social realizadas en el año 2017.
}


En el marco de este programa, Liliana Sinisi inició una línea de investigación centrada en el estudio de la diversidad cultural en escuelas de la Ciudad de Buenos Aires durante la década de los noventa. "Se interesó por el estudio de las diferencias, desigualdad, diversidad, discriminación. También aborda las representaciones y sentidos de los docentes, los procesos de alterización y estigmatización en las escuelas primarias" (L.C. 08/02/2018). Desde esta línea de abordaje, Liliana Sinisi se interesó por estudiar los usos del concepto "integración" en las escuelas y los procesos y prácticas que se derivan de la llamada "atención a la diversidad", particularmente de niños y niñas procedentes de diferentes etnias y naciones. Analiza que el "uso" del concepto "integración" sirve para dar cuenta de múltiples situaciones, tanto para explicar la forma de actuar de niños y niñas migrantes, para referirse a niños/as con diversas situaciones de discapacidad que deben estar en la escuela común, como para hacer referencia a las acciones que llevan adelante los/as docentes de educación especial (Sinisi, 1999). Desde el año 2010, estuvo vinculada al estudio de los denominados "circuitos escolares diferenciados" que atraviesan los niños y las niñas que son excluidos de la escuela común para ser derivados a escuelas que pertenecen al sistema de educación especial, situación que produce etiquetas, rótulos y tipificaciones. Durante esta investigación, observó que, desde el año 2004, el concepto de "inclusión” forma parte de las retóricas de políticas y programas socioeducativos que se vienen implementando en los últimos años en la Argentina y que es, además, "la categoría que por excelencia intenta definir un nuevo estado de situación” (Sinisi, 2010: 11).

En sus memorias, L.C. comparte que a partir del 2008 aproximadamente Liliana comenzó a dirigir proyectos de extensión universitaria: primero, del Programa de Voluntariado (Ministerio de Educación), y luego de UBANEX (de la UBA), radicados en el Centro de Innovación y Desarrollo para la Acción Comunitaria (Facultad de Filosofía y Letras de la UBA). Durante ese tiempo, coordinó una de las áreas de extensión de la facultad.

También, y vinculado a su pasado militante y a su compromiso político, los proyectos de extensión integraron una parte importante de su trayectoria porque, además, los concebía como espacios de formación en investigación, extensión y compromiso académico-político (P.M. 18/02/2018).

Una de las hipótesis de las cuales partió Liliana Sinisi en torno a las problemáticas que investigaba fue considerar que, a pesar de que la escuela no "reproducía de forma mecánica los procesos de discriminación y desigualdad" (Sinisi, 1999: 198) que encontramos en la sociedad, sí enmascaraba y naturalizaba estos mismos procesos en la conformación de una normalidad integradora de la "diversidad cultural [existente], cerrando de esta manera las posibilidades de comprensión de su dinámica histórica y social" (Sinisi, 1999: 189).

Es de esta forma que ella recupera la perspectiva etnográfica en educación desarrollada por Elsie Rockwell a partir de su experiencia en escuelas rurales mexicanas (de 1980 en adelante). Esta ha sido una importantísima contribución teórico-metodológica para comprender las tramas sociohistóricas en las que se despliegan las prácticas cotidianas de maestros/as, padres, madres, niños en los contextos escolares. Así, el enfoque etnográfico permite captar los múltiples contenidos de vastos procesos culturales. Además, ha incorporado la dinámica histórica en los espacios educativos relacionándolos con tramas más amplias de poder en complejos procesos de legitimación, apropiación, negociación y resistencia (Sinisi, 2013).

Liliana también trabajó para el Ministerio de Educación de la Nación; desde este espacio, habilitó las discusiones que venían forjándose a partir sus experiencias, su formación docente y como investigadora. 
Su ingreso al Ministerio fue en 2004... a partir de una recomendación de S. T. a quien por entonces era la coordinadora del Área de Investigación de la ex DINIECE (Dirección Nacional de Información y Estadística de la Calidad Educativa, del Ministerio de Educación, Ciencia y Tecnología de la Nación, tal como se llamaba en ese entonces) dado que buscaba introducir una 'mirada cualitativa' (P.M., 18/02/2018).

Entendemos que su experiencia laboral como antropóloga que trabaja en el Estado consistió en desnaturalizar lógicas enquistadas en este espacio de Trabajo. P.M. expresa:

\begin{abstract}
El ingreso del 'enfoque socioantropológico' supuso llevar adelante varias conquistas frente a los usos y costumbres del trabajo de campo que se realizaba en el Área de Investigación del Ministerio y de las investigaciones que se hacían y hacen a través de consultorías (...). Y, sobre todo, dar a entender que la 'demanda' de funcionarios y técnicos (que suele formularse en términos evaluativos: ¿cómo funciona tal dispositivo o... funciona bien o mal?) ‘habla del emisor’ (P.M. 18/02/2018).
\end{abstract}

De esta forma, desde el espacio de trabajo que compartía con otras compañeras antropólogas, retoman la perspectiva propuesta por Elena Achilli y problematizan que "lo que formulan/demandan es un problema de intervención y nuestra tarea consiste en construir un problema de investigación" (P.M. 18/02/2018). Nos señala L.C. que muchos de los aportes que hizo desde su función en el Ministerio se publicaron en la Serie Educación en Debate, que se encuentra disponible en formato on line.

Finalmente, resta por decir que, pese a residir en Buenos Aires, Liliana Sinisi mantenía siempre contacto por mail con compañeras de la Red de Investigación Antropología y Educación (RIAE) de la que formaba parte, y que tuvo su primer encuentro en Huerta Grande (Córdoba) en 2010 con la modalidad de seminario-taller que buscaba abordar problemáticas en torno a "La Antropología de la Educación en Argentina. Problemas, Prácticas y Regulaciones Políticas”. Finalmente, E.A. nos menciona que algo que la mantenía entusiasmada era la tesis que estaba haciendo para obtener su doctorado en Antropología de la UBA.

\title{
Nuestras problemáticas de investigación atravesadas por los inter- cambios con Liliana Sinisi
}

Luego de este recorrido por su trayectoria, consideramos que la línea de trabajo desarrollada por Liliana Sinisi, acerca de los procesos de "integración-inclusión/exclusión” escolar supone una de las temáticas más abordadas por esta investigadora. En este sentido, nos interesa presentar nuestros trabajos de campo realizados en el periodo 2008-2013 acompañados por esta investigadora a través de sus aportes, análisis y problematizaciones. En el caso de una nosotras, mediante la dirección de tesis; y en el otro, desde la lectura y el intercambio vía mail a raíz de formar parte de la RIAE.

Las problemáticas de investigación están vinculadas a las experiencias de labor docente relacionadas con niños y niñas con "Necesidades Educativas Especiales derivadas de la discapacidad" (de ahora en adelante, "NEE”) que transitan la escuela de educación "común". Nuestras investigaciones se realizaron en un Centro de Servicios Alternativos y Complementarios de la Ciudad de Sarmiento, Chubut y en una escuela confesional privada de la zona centro de la ciudad de Rosario.

Liliana Sinisi nos propuso analizar las tensiones y contradicciones que se construyen en torno al complejo entramado de los procesos de "integración-inclusión/exclusión" que atraviesan la experiencia escolar de niños, niñas y jóvenes que, por ser considerados 
"sujetos de la educación especial", ven cercenado su derecho a acceder/permanecer en la escuela "común".

En el siguiente apartado, presentaremos algunos de los conceptos y categorías teóricas y sociales que nos aportó Liliana Sinisi para problematizar nuestros trabajos de campo.

\section{Presentación de las investigaciones}

Consideramos oportuno aclarar que la iniciativa de este escrito surgió luego de un encuentro en un seminario cursado en la Universidad Nacional de Córdoba, dictado por María Rosa Neufeld en el marco del Doctorado en Estudios Sociales de América Latina. Allí nos encontramos las autoras de este trabajo y, frente a las lecturas propuestas por el seminario, decidimos retomar aquellas producciones de Liliana Sinisi. Las discusiones que allí se produjeron nos llevaron a retomar nuestros antiguos trabajos de campo; y a partir de esto, pensamos que sería importante homenajear su trayectoria en tanto referente clave, no sólo de nuestras investigaciones, sino de muchas otras, construidas por investigadores/as de distintos campos de estudio.

Por otra parte, consideramos necesario que los aportes que Liliana Sinisi realizó respecto de cómo pensar las formas que adoptan los procesos de desigualdad social en las escuelas no se limita a la problemática o análisis de "integración-inclusión" escolar de niños con "NEE" o "discapacidad", como veíamos al presentar su trayectoria. También ayudan a pensar lo educativo en un sentido amplio, incluso más allá de las relaciones escolares, en distintos niveles y escalas de análisis, así como también rastreando las huellas que encubren aquello que ella menciona como una forma de racismo. Sin embargo, nos pareció significativo poder anclar esto en las experiencias de nuestros intercambios, lo cual da cuenta de la relevancia y vigencia de su mirada, tanto en nuestras investigaciones, como en el campo educativo en un sentido amplio.

Otra aclaración que hace a la presentación de este escrito estaría vinculada a que decidimos "entrecomillar" diferentes conceptos que aparecen a lo largo del texto, con la intención de ponerlos en duda y/o tensionar los distintos sentidos con los que son utilizados. Algunos de estos conceptos son "Necesidades Educativas Especiales", "integración", "inclusión", "adaptaciones", "acompañamiento". Sostenemos que resulta necesario no dar por sentado que, cuando hablamos de "integración", todos/as hablamos de lo mismo; que cuando leemos en una política pública "inclusión”, no necesariamente se refleja el mismo sentido que una/un docente trabaja desde el aula. También, nuestro interés radica en poder poner en cuestión estos conceptos y llenarlos de los múltiples sentidos, poniendo en juego los matices que estos encarnan, sin naturalizar aquello que busca ser institucionalizado. Algunas de nuestras preguntas son: ¿quiénes son estos/ as llamados/as niños, niñas y jóvenes con "NEE"? ¿Quiénes o qué define quién es un niño o niña con "NEE"? Cuando hablamos de "integración-inclusión-adaptación" escolar: ¿qué nos dicen los planes, programas, las políticas públicas?, ¿qué nos dicen los docentes al respecto?, ¿cómo viven las familias estos procesos? Las comillas, entonces, nos ayudan a mantener presente esta problematización y tensión constante a lo largo de nuestro proceso de escritura con la intención de poder proporcionar en la lectura la tensión constante de estos procesos.

A continuación, haremos una presentación de nuestros trabajos de campo, cómo conocimos a Liliana y los aportes y discusiones que fueron nutriendo nuestras investigaciones. 


\section{Los Centros de Servicios Alternativos y Complementarios en los procesos de "derivación"}

En el año 2009 conocí a Liliana Sinisi en un seminario de posgrado que ella dictaba junto a otras profesoras en la Maestría de Psicología Educacional de la UBA. En el marco de este seminario, Liliana propuso desde la antropología social —más precisamente desde el enfoque etnográfico en educación (Rockwell, 1987) — problematizar algunas categorías tales como "Diferencia", "Diversidad" y "Desigualdad", con la intención de abrir la discusión respecto de los "usos" de la "diversidad sociocultural" y de las formas en que estos "usos" se entrelazan con categorías como la "pobreza" y la "desigualdad" en los contextos educativos. También, incluyó en estas problematizaciones los debates en torno a las polémicas que en ese momento existían en relación con el concepto de "educabilidad"3 y de "resiliencia".

Durante la cursada del seminario, Liliana nos proponía desde la etnografía, ir problematizando distintos procesos sociales desde un análisis que concebía al sujeto en relaciones y en contextos más amplios, poniendo en tensión nuestros propios enfoques disciplinares con los cuales pensábamos y analizábamos la realidad educativa y escolar.

En una de sus clases, compartió algunos de los avances de su investigación para la tesis doctoral - que se encontraba en proceso de escritura- sobre "los circuitos escolares diferenciados" que atraviesan los niños y niñas que son excluidos de la escuela común para ser derivados a escuelas que pertenecen al sistema de educación especial, lo que produce etiquetas, rótulos y tipificaciones (Sinisi, 2010). A diferencia de la mayoría de las investigaciones que, desde diferentes campos de estudio - fundamentalmente desde la psicología y la pedagogía- suelen abordar las relaciones entre la educación común y la educación especial, que centran el foco de sus análisis en los niños y niñas que son derivados a educación especial por problemas de aprendizaje, cognitivos, de conducta, hiperactividad y otros muchos "síndromes" con los que son etiquetados, en esta investigación, Liliana proponía un enfoque diferente. En este sentido, fueron claves el análisis y la crítica que planteó en relación con las investigaciones que abordaban esta temática desde la resolución de "casos" de los niños y niñas que han sido derivados y/o integrados en los diferentes circuitos educativos. Al decir que se trabajaba sobre "casos", Liliana sostenía que se trataba de un abordaje que partía del niño y su singularidad incluyéndolo en las redes familiares, pero muy pocas veces en niveles locales e históricos que estructuran tramas más amplias y complejas, como pueden ser los procesos de desigualdad social (Sinisi, 2013). Este aporte resultó significativo para lo que en ese momento estaba construyendo como problema de investigación para mi tesis de Maestría, respecto de los procesos de "derivación" de educación "común" a educación "especial" de niños y niñas que transitaban el primer ciclo de la escuela primaria. Por este motivo, le propuse a Liliana Sinisi ser mi directora de Tesis de Maestría. Desde ese momento, mantuvimos distintos encuentros presenciales durante mis viajes a Buenos Aires, y también mediante largas charlas por Skype y correos electrónicos. En el año 2011 Liliana aceptó la invitación para que conociera mi ciudad de origen, Comodoro Rivadavia. Aprovechando su visita, organizamos un seminario de posgrado que dictó junto a Laura Santillán en la Facultad de Humanidades y Ciencias Sociales de la Universidad Nacional de la Patagonia San Juan Bosco. A este seminario no sólo asistieron compañeros y compañeras del ámbito académico universitario, sino también muchos/ as docentes de escuelas de educación común y de educación especial que la conocían por sus diversas producciones. Un rasgo interesante de Liliana, y por el cual considero que su trayectoria ha sido muy significativa, fue su interés y apertura que dedicaba en todo momento al análisis de la realidad escolar, lo cual la llevaba a participar y presentarse en talleres con docentes en distintas escuelas del país, congresos y jornadas vinculadas a lo educativo, muchas de ellas específicas del campo de la educación especial, como lo son las jornadas de RUEDES (Red Universitaria de Educación Especial).
2. En este apartado se presenta la investigación desarrollada por Cristina Pereyra, en el marco de la tesis de maestría en Psicología Educacional UBA, "Las derivaciones de educación común a educación especial de niños y niñas que habitan en contextos de pobreza y que transitan el primer ciclo de la educación primaria", cuya directora fue la Lic. Liliana Sinisi.

3. Neufeld y Thisted plantean que "educabilidad" es un concepto que determinados autores parecen querer imponer para reemplazar, con sentidos algo modificados, conceptos anteriores. Lo encontramos en textos recientes de sociólogos de la educación; los que lo usan trazan vinculaciones con sus antecedentes: "destacamos una vinculación indiscutible entre 'educabilidad' y 'resultados esperados del proceso de socialización', que seguramente hubiera sido el concepto evocado en años anteriores" (Neufeld y Thisted, 2004: 86).

4. Neufeld y Thisted discuten el concepto "resiliencia" planteando que "en las definiciones y descripciones acerca de la resiliencia, los aspectos conflictivos no son evidentes" (Neufeld y Thisted, 2004:93), es decir, que este concepto no plantea las causas que llevaron a los sujetos a estas situaciones "desfavorables". 
En estos encuentros, sus intervenciones marcaban un antes y un después en las distintas discusiones, de las cuales tuve la posibilidad de participar, siempre con una impronta que intentaba debatir en torno a las tramas naturalizadas de la cotidianeidad escolar.

La investigación que presentamos en este apartado se desarrolló en la ciudad de Sarmiento, provincia de Chubut durante los años 2011 y 2013. El foco de esta investigación se planteaba los procesos de "derivación" de educación "común" a educación "especial" de niños y niñas que transitaban el primer ciclo de la escuela primaria, entendiendo que en ellos se ponían en juego sentidos y prácticas docentes de maestras/os de educación especial y de educación común, equipos directivos, equipos interdisciplinarios de orientación de educación especial, grupos familiares, entre otros/as actores educativos y escolares. En la construcción de esta problemática de investigación, resultó clave el aporte de Liliana respecto de la necesidad de poner en tensión las relaciones entre la educación común y la educación especial a partir de las políticas educativas sobre la inclusión e integración escolar propuestas por las reformas neoliberales del Estado y cómo estos procesos en las prácticas educativas generaban movimientos contradictorios que, en el fondo, terminan configurando procesos de exclusión y segregación escolar. Este análisis supone entender las tramas escolares cotidianas, sin perder de vista los procesos más amplios que las atraviesan y las constituyen. Desde esta perspectiva, partimos de considerar la escuela no en forma aislada, sino como una institución que adquiere sentido si se la considera en vinculación con los procesos y relaciones contextuales que la conforman y, al mismo tiempo, la trascienden (Achilli, 2010).

Durante el trabajo de campo, se realizaron entrevistas en profundidad, observaciones participantes y encuentros informales con maestras de educación "especial" que trabajaban en una escuela "especial"; con maestras de educación "común" de niños y niñas por los cuales se "solicitaba" una "derivación" a educación "especial" en dos escuelas primarias; con directoras y supervisoras de las escuelas de educación "común" y educación "especial"; y con las integrantes de los equipos interdisciplinarios de educación "especial" (psicopedagoga, licenciada en Ciencias de Educación, trabajadora social y fonoaudióloga). También se accedió a la lectura de legajos de alumnos/as y documentación involucrada en el proceso de derivación a educación especial, en la que se incluyen documentos, normativas, notas y/o circulares, proyectos educativos institucionales.

En las escuelas de educación común se conoció a las maestras de apoyo a la inclusión que concurrían a "acompañar" en sus procesos de aprendizaje a niños y niñas con necesidades educativas derivadas de la "discapacidad". Estas maestras de apoyo a la inclusión pertenecían a los Centros de Servicios Alternativos y Complementarios (CSAyC), instituciones creadas a principios de los noventa en la provincia del Chubut que desde su origen dependen de la Dirección General de Educación Especial del Ministerio de Educación Provincial. El Centro de Servicios Alternativos y Complementarios de la ciudad de Sarmiento se creó en el año 2005.

Las maestras de apoyo a la inclusión que conforman estos centros realizan su tarea docente en escuelas de nivel inicial, primaria y secundaria, "acompañando" procesos de inclusión educativa de niños y niñas que en situación de "discapacidad" "presentan" "problemas de aprendizaje" en las escuelas de educación "común" a las que concurren. Las docentes de educación común solicitan la intervención de las maestras de apoyo a la inclusión, para que "acompañen" a aquellos/as niños y niñas que presentarían "problemas" en su aprendizaje vinculados a "necesidades educativas especiales" relacionadas con las características de las familias, situación social o económica de los

5. Información extraída de los pedidos de las docentes de educación y de las entrevistas realizadas a docentes de educación común y a maestras de apoyo a la inclusión. niños/as, como así también, a aquellos niños y niñas que tiene diagnósticos o informes de especialistas de salud. ${ }^{5}$ 
Por otro lado, en la escuela de educación "especial” se pudo conocer que en los últimos años "no habían ingresado" a la escuela niños y niñas "derivados" de las escuelas de educación "común". Según las maestras y las directoras de esta escuela, las razones por las cuales no habría "ingresos" estarían vinculadas con la creación del Centro de Servicios Alternativos y Complementarios, que de alguna manera a través de sus intervenciones en las escuelas de educación común "frenaba” el ingreso a la escuela especial.

A partir de la lectura de legajos de niños y niñas que habían ingresado a la escuela de educación "especial", se desprende que todos/as ellos/as lo habían hecho antes de la creación del Centro de Servicios Alternativos.

Por su parte, en las escuelas de educación “común”, las maestras y las directoras continuaban "sosteniendo" la necesidad de las "derivaciones" de los niños y las niñas con "necesidades educativas especiales" a "educación especial", entendiendo por "derivaciones" a los "pedidos" de "intervención" de las maestras de apoyo a la "inclusión" del Centro de Servicios Alternativos y Complementarios (CSAyC).

Durante el trabajo de campo se pudo conocer que las derivaciones a educación especial estarían identificadas por dos procesos diferentes. El primero de ellos, vinculado al pase de un alumno/a de una escuela de educación común a una escuela de educación especial. El segundo estaría representado en la incorporación de alumnos/as a los denominados proyectos "inclusión escolar" dependientes de educación especial, y que forman parte del servicio de apoyo que prestan los CSAyC.

Por último para cerrar este apartado, considero que el aporte de Liliana a esta investigación, y que dejó una huella significativa, fue la perspectiva relacional (Achilli, 2005) que me propuso construir, lo que me permitió animarme a realizar análisis relacionalmente, tanto de los sentidos como de las prácticas sociales en contextos de desigualdad social y económica.

\section{Rocamadur: una escuela confesional privada de la zona centro de la ciudad de Rosario ${ }^{6}$}

Conocí a Liliana en el segundo encuentro de la RIAE que se organizó en Carcaráes. Este encuentro había sido organizado por el equipo de Antropología y Educación del Centro de Estudios Antropológicos en Contextos Urbanos (CEACU) que pertenece a la Universidad Nacional de Rosario (UNR). Yo había comenzado a participar del mismo en octubre de 2011 por ser tesista de Elena Achilli (Directora de mi investigación) y Verónica Greca (Codirectora). Elena, quien ya me había dado el mail de Liliana, me dijo que me la iba a presentar para que pudiéramos realizar intercambios por mail. Elena resaltaba lo accesible que era Liliana pero, pese a sus palabras, yo no me animaba a iniciar este intercambio. Recuerdo que ese encuentro fue muy significativo para mí, porque entre las paredes de la casa que nos alojaba en Carcaráes descubrí que el mundo académico podía tener espacios de trabajo que contemplasen lo afectivo sin perder el rigor y la mirada crítica que las investigaciones merecen, pero generando espacios amables y humanos de intercambio. Luego de la presentación y apertura de los espacios de trabajo que tuvo lugar el primer día, Elena me llamó y me dijo que me iba a presentar a Liliana. Camino de la sala de reuniones al hall principal, recuerdo haberme sorprendido: le estaba poniendo cara a "la bibliografía obligatoria", a aquellas personas que me invitaban a pensar/problematizar/desnaturalizar los sentidos de las realidades sociales, de las rupturas y continuidades de los procesos, de las categorías. Recuerdo que con Elena nos paramos frente a dos mujeres que me parecieron extremadamente altas; con el tiempo me enteraría por los compañeros de la RIAE que las llamaban "hermanitas": Liliana Sinisi y Elisa Cragnolino (actual directora de mi beca
6. Este apartado presenta la experiencia de Macarena Romero Acuña durante intercambios mantenidos con Liliana Sinisi a partir del proceso de escritura de la tesis para la obtención del título de licenciada en Antropología de la Universidad Nacional de Rosario. 
doctoral, proyecto que comencé a pensar con la dirección de Liliana y la codirección de Elena Achilli a principios de 2014). Conocer a Liliana significó alejar ese fantasma de construcción desde la formalidad de la asimetría docente-estudiante. Y creo que esto último es el gran capital que tienen tanto la RIAE como los equipos de investigación que la conforman: se ponderan las construcciones críticas de conocimiento y los procesos investigativos tanto como el cuidado de las relaciones afectivas y humanas. Luego de este encuentro en Carcaráes, iniciarían los intercambios por mail y algunos viajes a Buenos Aires cuando empezamos a pensar el proyecto doctoral para presentar al Consejo Nacional de Investigaciones Científicas y Técnicas.

El trabajo de campo de mi tesis de grado en la Licenciatura en Antropología se desarrolló desde el año 2008 al 2012, con docentes de educación "común" y docentes de educación especial "integradoras" de una escuela confesional privada en la ciudad de Rosario, provincia de Santa Fe. El foco de interés de la investigación se centraba en analizar las formas que adopta el trabajo docente en su relación con los procesos de "integración" de los niños y las niñas con "NEE" en una escuela confesional privada del centro de la ciudad de Rosario. Para esto, decidimos realizar entrevistas en profundidad tanto con "docentes especiales", como con "docentes de grado", docentes de "apoyo a la integración" y "docentes integradoras".

La intención a lo largo del trabajo de tesis fue narrar sus recorridos, los sentidos y sus prácticas como docentes en relación con los niños con "NEE" de la escuela Rocamadur. Preguntarnos qué significado le dan algunas de las docentes a la "integración/inclusión" para entender, de acuerdo con sus construcciones, quiénes son estos niños con "NEE" y cómo ellas organizan su trabajo docente.

Algo que quebró y restructuró el trabajo de campo fue el “Taller de Integración” propuesto por la docente Sabina en principio, y continuado por la docente Talita. Inicialmente, Sabina, por una cuestión de organización, planteó dar un taller para aquellos niños con "NEE" que asistían a la Escuela Rocamadur y a quienes ella hacía seguimiento. Los motivos tenían que ver con que al colegio cada vez asistían más niños que precisaban de los "servicios" que ella brindaba en la institución como maestra "especial" y la "cantidad" excedía su capacidad de acompañamiento. Un segundo motivo consistía en que "yo también estoy como que en una necesidad interna de empezar a trabajar de una forma más independiente y no estar siempre de alguna forma bajo la sombra de un docente de grado, por decirlo de alguna forma" (Sabina, Rosario 20/08/08).

El taller estaba pensado para todos los niños con dificultades motrices o discapacidades, y también estaban incluidos los chicos con problemas, por ejemplo, de "adaptación" a la escuela.

Sí están incluidos, chicos violentos que es lo que más vengo viendo. En lo que es la integración, el down, el ciego, el sordo, se están viendo otras problemáticas, y esos chicos con problemas del orden que uno llama de la subjetivación, con problemas de conducta, de socialización, obviamente que... la propuesta yo la empecé a gestar en función de esos chicos (Sabina, Rosario 20/08/08).

De esta forma, los aportes y los intercambios con Liliana (siempre guiados y acompañados por Elena Achilli y Verónica Greca) fueron centrales para poder problematizar, a la vez que pensar y tensionar, aquello con lo que nos encontrábamos en el trabajo de campo. Liliana Sinisi denomina "homologización paradójica" (Sinisi, 1999: 212) a las situaciones que convalidan "deficiencia cultural y social" con "deficiencia intelectual". De esta manera, se legitiman procesos de derivación a educación "especial" de muchos niños/as que, por sus supuestas dificultades vinculadas a contextos de pobreza o de 
dificultad dentro del aula, terminan transitando su escolaridad en las escuelas de educación "común" como sujetos de la educación "especial".

\section{"Cada vez son más..." Entre la educación "común” y la educación "especial"}

"yo no entiendo muy bien qué está pasando, pero de repente una entra al aula y se encuentra con que, o antes no estaban, o no sé por qué estos chicos ahora cada vez son más" (Olivia, Rosario, 17/06/2008).

Consideramos que la cita transcripta expone algunos procesos escolares por los cuales Liliana Sinisi planteaba una gran preocupación y se constituían en el foco de sus investigaciones. Fundamentalmente, aquellos en los que niños y niñas son excluidos de la escuela común para ser derivados a escuelas que pertenecen al sistema de educación "especial", lo que produce "circuitos escolares diferenciados" que, al decir de Liliana Sinisi configuran y reconfiguran la experiencia escolar infantil, en tanto en los diferentes circuitos se producen experiencias formativas singulares de aprendizaje que pueden marcar la trayectoria futura de estos/as niños y niñas (Sinisi, 2010).

Este análisis se desprende de su tesis doctoral, "Entre la educación común y la educación especial: los circuitos escolares diferenciados y el problema de la integración. Un estudio histórico-etnográfico en contextos de diversidad y desigualdad social", que se encontraba en proceso de escritura, para lo cual realizaba trabajo de campo en escuelas de recuperación de la ciudad de Buenos Aires.

Algunos de los avances de esta tesis fueron presentados en distintas publicaciones y presentaciones en encuentros académicos. Nos interesa recuperar algunos de ellos porque consideramos que son análisis clave para el estudio de los denominados procesos de "integración" y de "inclusión" escolar.

En este sentido, consideramos que uno de los importantes aportes estaría vinculado al posicionamiento teórico-metodológico desde el cual investigaba, a partir de un enfoque etnográfico en educación.

Desde esta perspectiva, sostenemos junto con Liliana Sinisi que el enfoque antropológico o la etnografía educativa permiten comprender la complejidad de la trama de sentidos de los sujetos que participan de los denominados procesos de "integración" e "inclusión" educativa - docentes, niños, familias, especialistas de gabinete etc.— en tanto disputan los significados sobre lo que se entiende que puede ser la experiencia escolar "inclusiva” (Sinisi, 2010).

Por otra parte, entendemos como fundamental el planteo que realizó Sinisi respecto del "uso" del concepto de "inclusión". Esta autora sostiene que aparece fuertemente en el nivel estatal, a partir del año 2004, en los fundamentos de las políticas socioeducativas, en las que "derecho" e "inclusión" aparecen como categorías dominantes (Sinisi, 2010). Asimismo, plantea que las prácticas que construyen los sujetos sociales en la cotidianeidad de las escuelas en torno a los procesos de "inclusión" educativa adquieren particularidades diversas, entendiendo que "es el marco teórico en conjunto con el contexto en el que se produce y los usos que se realizan de ella, lo que va a definir sus significados, nunca acabados y siempre en tensión" (Sinisi, 2010: 11).

Estos aportes de Liliana en nuestras investigaciones nos permitieron, durante el trabajo de campo, interrogarnos acerca de quiénes son estos/as niños, niñas y jóvenes "incluidos" e "integrados" en las escuelas educación "común"; y cómo son las prácticas educativas 
que se construyen en torno a estos niños y niñas de la educación "especial". También nos preguntamos: ¿cómo son las prácticas docentes y los sentidos que construyen las/ os maestras/os de educación "especial" y de educación "común" que realizan su trabajo docente en estos procesos denominados de "integración" y de "inclusión" educativa?

Respecto de la investigación realizada en Sarmiento, las maestras de apoyo a la inclusión sostenían que la escuela más "carenciada" de la ciudad sería la que presentaría "mayor demanda" y donde existiría más cantidad de niños y niñas "incluidos" (niños y niñas que están inscriptos en el proyecto de inclusión educativa del CSAYC).

En el marco de una investigación realizada entre los años 2000 y 2002, Liliana Sinisi y Paula Montesinos analizan las relaciones entre las representaciones "sobre la niñez en contextos de pobreza", las prácticas institucionales con ella relacionadas y su vinculación con los circuitos de inclusión-exclusión social y educativa. Estas investigadoras realizaron un aporte interesante respecto de cómo la infancia en contextos de pobreza es objeto de una educación diferente/desigual que reciben de las escuelas donde los niños/as transitan su escolaridad, y cómo está dimensión también contribuye a un presente y un futuro de frustración y exclusión (Sinisi y Montesinos, 2003).

Respecto de las prácticas docentes que realizaban las maestras de apoyo a la inclusión del CSAyC con los niños y niñas por los cuales "intervenían", muchas de estas solían partir de la "necesidad" y "patología" que estos presentaban, definiendo sus prácticas docentes en relación con ello. En general, se centraban en la realización de propuestas "diferenciadas", como actividades con contenidos del nivel inicial, entre otras. A su vez, la mayoría de los niños y niñas que estaban "incluidos/as" no necesariamente presentaban una situación de discapacidad, sino, como se planteó anteriormente, que sus "necesidades" estarían vinculadas a situaciones de desigualdad social y económica. De esta manera, podemos problematizar respecto de los sentidos que se construyen en torno a estas prácticas, los cuales estarían vinculados a procesos de estigmatización y discriminación, y que desde un lugar de "déficit" y "patología" naturalizarían las desigualdades sociales y económicas.

En el caso de la Escuela Rocamadur; invitó a pensar por qué surgía en las narrativas de las distintas docentes el: "cada vez son más" los niños con "NEE". Eje articulador que lleva a la construcción del Taller de Integración por parte de las docentes especiales, pero que a su vez es expresado como malestar o desde una pregunta por otros docentes de la institución. Para esto, nos fue necesario historizar el proceso que lleva a la construcción de estos niños llamados con "NEE". Nuestra pregunta central fue: ¿quiénes son los niños con "NEE"?, ¿cómo llegan a la escuela?, ¿cómo construye la escuela a estos niños?

En relación con la investigación que desarrolló Liliana Sinisi respecto del "uso" del concepto "integración", la autora sostiene que este sirve para dar cuenta de múltiples situaciones: para explicar las diferencias en el aprender, en los modos de actuar de aquellos que no responden a las expectativas de la escuela, ya sean migrantes, con experiencia de pobreza, con discapacidad u otros, como así también para justificar las acciones que llevan adelante los docentes de educación especial en las escuelas comunes (Sinisi,1999). Situación que, como ya mencionamos, la autora denomina "'homologización paradójica', que se realiza entre los procesos de integración de la diversidad y aquellos otros referidos a la incorporación de la escuela común de los niños con discapacidades físicas o necesidades educativas especiales" (Sinisi, 1999: 212).

Los aportes que presentamos de Liliana nos permitieron ir problematizando nuestros trabajos de campo, comprender los procesos y dinámicas que se ponían en juego en torno a las prácticas y sentidos que construían los sujetos sociales respecto de los procesos de inclusión educativa en nuestras investigaciones. 
En este sentido, sostenemos que el "cada vez son más” es una disputa de sentidos históricos, políticos, económicos y morales que muestran, con distintos matices, procesos de diferenciación social que se traducen en "integraciones" e "inclusiones", pero también en exclusiones y en formas del racismo. Conocimos, durante el desarrollo de nuestros trabajos de campo, que muchos de los niños, niñas y jóvenes que se encuentran transitando la escuela "común" son "derivados" a los denominados proyectos de "integración” y de "inclusión" escolar que dependen de educación especial y se constituyen en "circuitos escolares diferenciados" (Sinisi, 2012), cuyas problemáticas en el aprender estarían vinculadas con su situación social, económica, de pobreza. Situación que termina naturalizando procesos de desigualdad social y económica.

\section{Problematización de procesos contradictorios de integración- inclusión/exclusión escolar}

Como señalamos, Liliana Sinisi advirtió respecto de las contradicciones, tensiones y conflictos que suponen las relaciones entre los denominados procesos de "inclusión educativa" y las políticas socioeducativas, especialmente de aquellas que en contextos de políticas neoliberales ponían énfasis en la incorporación de niños, niñas y jóvenes a los distintos niveles del sistema educativo (Sinisi, 2012). En este planteo, Sinisi sostuvo la necesidad de analizar las prácticas y los sentidos que construyen los actores sociales en la cotidianeidad de la escuela respecto de la denominada "inclusión educativa", así como también alertó sobre los "usos" y las apropiaciones siempre heterogéneas que estos/as sujetos sociales ponen en juego, en las cuales se producen nuevas configuraciones culturales en torno a la experiencia escolar.

Al decir de Rockwell y Ezpeleta y Sinisi, la relación entre el Estado y los sujetos adquiere contenidos particulares en su existencia cotidiana y, en tanto tales, históricos, al tiempo que en dicha relación operan múltiples mediaciones que no pueden ser definidas a priori (Rockwell y Ezpeleta, 1985; Sinisi, 2008). Desde la perspectiva de estas autoras, se sostiene que los singulares sentidos construidos por los actores escolares resignifican las orientaciones presentes en las políticas y programas en su fase de implementación, con lo cual se producen modificaciones en sus prescripciones y contenidos originales. Según Sinisi, "En realidad, es en ese campo de mediaciones donde se juegan los contenidos y modalidades concretas que asumen las diferentes políticas y programas estatales referidos, en este caso, a la inclusión" (Sinisi, 2012: 61).

Respecto de la investigación desarrollada en el Centro de Servicios Alternativos y Complementarios, nos encontramos con que, tanto para las maestras de las escuelas de educación "especial", como para las maestras de apoyo a la inclusión el Centros de Servicios Alternativos y Complementarios, el "número" de niños y niñas "identificados" como matrícula de educación especial se habría incrementado, debido al ingreso de niños y niñas al Proyecto de "inclusión educativa” dependiente del Centro de Servicio Alternativo y Complementario de la ciudad de Sarmiento. Al depender del área de Educación Especial, sitúa a los niños/as "destinatarios" de sus intervenciones en la paradójica situación de la "doble matrícula" de educación "especial” y de educación "común". Por otra parte, las maestras de las escuelas de educación especial entendían que los "ingresos" a educación especial habían descendido, debido a que no existían “derivaciones” de niños y niñas a estas escuelas.

Podríamos plantear que estos/as niños y niñas “integrados" o "incluidos” continúan siendo los "excluidos" del espacio escolar. Situación que Liliana Sinisi reconoció al sostener que se produce un "proceso complejo y contradictorio de integración/exclusión que termina, por estigmatizar y cargar de prejuicio a aquellos que son ubicados en el lugar del 'diferente' al interior del sistema educativo' (Sinisi, 1999: 223). 
En este sentido, se podría plantear que las intervenciones del CSAYC, en situaciones en que las escuelas de educación "común" ponen en duda las posibilidades de aprender de los niños y niñas debido a su situación "social" y "económica", se ponen en tensión con las políticas socioeducativas de "integración-inclusión" educativa que intentan "incluirlos/as".

Yo me recibí en el 2003, no se hablaba de inclusión, se hablaba de integración. Y por ejemplo esta chica que te comentaba antes que se recibió en 1988, ni siquiera de integración. ¿Me entendés? Y ahora estamos en el 2012, nueve años después y estamos en el paradigma de la inclusión (Lucía, Rosario 19/10/2012).

La propia historia de los sujetos, así como también sus experiencias y recorridos dentro de lo que es la educación, y más concretamente en relación con lo que es la educación "especial", da forma al trabajo que dichos sujetos realizan. Las perspectivas acerca del trabajo, la valoración que tienen de él y la forma de llevarlo a cabo se encuentran relacionadas a las experiencias escolares mismas que estas docentes vivieron. Y no estamos haciendo referencia a un mero reproductivismo de la experiencia escolar. Nos referimos a que las formas en que las docentes aprehendieron sus experiencias escolares hacen o forman parte de cómo "cocinan" su trabajo como docente. De ahí que no hay una sola forma de comprender el proceso de "integración-inclusión" escolar llevado a cabo por las docentes dentro de la institución. En este sentido fue que, en la escuela Rocamadur, pudimos ver cómo tanto Sabina como Lucía, desde los talleres al acompañamiento dentro del aula, dejaron su impronta; en ellos, si bien el trabajo que realizaron desde el puesto otorgado por la institución fue el mismo, a su vez, no lo fue. Sus huellas marcaron encuentros y desencuentros en el cómo llevar adelante el proceso de "integración" escolar.

Sus recorridos nos hacen pensar en cómo la escuela es un todo dentro de un todo mayor que constantemente la atraviesa, ya sea desde lo histórico, que se plasma en el aula; en la normativa, que se manifiesta en, parafraseando a muchos docentes: "las filas con una baldosa de distancia entre alumno y alumno"; ya sea en aquello experimentado dentro de la propia experiencia escolar vivida por los docentes, aquello que traen consigo.

También atraviesan estas experiencias las condiciones materiales y socioeconómicas que atraviesa la escuela. Como también aquellas que viven los alumnos que a ella concurren y sus familias. Condiciones sociohistóricas y materiales en las que se encuentran las docentes y directivos y en las que actualmente estos viven. Achilli plantea que

la vida escolar se va constituyendo con mucho de las prácticas y relaciones de las familias que las circundan y, viceversa, la vida familiar incorpora muchas de las relaciones y prácticas escolares experimentadas y/o representadas por sus integrantes (Achilli, 2010: 82).

De esta forma, pensar a la escuela desde una perspectiva relacional dialéctica nos ha ayudado a dar forma a estas relaciones que se entraman tejiendo la dinámica y la vida de la escuela y del trabajo docente. Esta clave dialéctica y en constante "ida y vuelta" con la realidad social, también ha posibilitado pensar y tensionar el trabajo docente. Lo primero que aparece, entonces, es la necesidad de "desnaturalizar", interrogar y repensar aquellas prácticas institucionales y pedagógicas que sostienen y refuerzan las bases de una escuela para "los que están en condiciones", para "los que pueden o quieren aprender", para "algunos" (Montesinos, Sinisi y Schoo, 2009). Tratamos de interpelar algunas de las estructuras que han cimentado la formación de los propios docentes para poder dar cuenta de la complejidad del entramado que atraviesa a sus prácticas. 
Creemos indispensable cerrar esta reflexión en torno a los aportes de Liliana Sinisi retomando sus palabras cuando dice:

Acuerdo totalmente, con el enunciado que Eduardo Menéndez realizó hace ya algunos años y que a mi entender ha recobrado una nueva vigencia: 'El racismo no es solamente una cuestión de segregar negros u odiar judíos; el racismo debe ser referido a las formas de las relaciones sociales y culturales que implican negación, discriminación, subordinación, compulsión y explotación de los otros en nombre de pretendidas posibilidades y disponibilidades, ya sean biológicas, sociales o culturales. Toda relación social que signifique cosificar a los otros, es decir negarles categorías de personas, de igual; toda relación que permita la inferiorización y uso de los otros es racismo' (Menéndez, 1968). Éstas son las cuestiones de las que todos debemos hacernos cargo (...) no debemos olvidar que la escuela forma parte de la sociedad y que los modelos económicos, políticos y culturales en los que abreva el neoliberalismo conservador, en la medida en que hegemonizan los discursos en torno a la alteridad, propician y refuerzan la construcción de sentidos ligados a la estigmatización y racialización. (...) La escuela sigue siendo un espacio para la transformación social (Sinisi, 1999: 228-229).

\section{A modo de cierre...}

Dar clases... formar... le insumía gran parte de su tiempo... pero era una tarea que la gratificaba... formar a los 'nuevos' (como dicen las pedagogas)... tanto que las múltiples docencias (más allá de la necesidad de laburo que todos tenemos) le quitaban tiempo y energía para poder producir más en torno a su tema de investigación... Sin embargo, estoy convencida que las actividades de docencia, conferencista, panelista, etc... tan profusas!!! Fueron momentos muy importantes para su propia producción intelectual (P.M., 18/02/2018).

Este ha sido un primer acercamiento al entrecruzamiento de escrituras y de campos. Creemos que nos queda por seguir profundizando este análisis en términos de políticas diferenciadas entre provincias; continuidades y rupturas en los procesos formativos de las docentes y de los estudiantes; también en términos de discusión teórico-metodológica entre campo y teoría. Sin embargo, no quisimos dejar de presentar esta breve reseña que aporta a seguir pensando y problematizando los procesos de "integración" y de "inclusión" educativa; a la vez que es una apuesta colectiva a poder pensar y tensionar distintas investigaciones, experiencias y recorridos a raíz de transitar una misma problemática de estudio.

Para nosotras ha sido un placer la escritura de este homenaje, porque escribir acerca de Liliana nos ha permitido pensarla y recordarla de distintas maneras y en distintos momentos de su vida y de las nuestras.

Nos pareció muy importante resaltar su trayectoria académica, pero también aquellas formas que ella tenía de transmitir el oficio de la antropología: mirada tenaz, desafiante y crítica de la realidad que no perdía de vista las relaciones y los procesos afectivos que entrecruzan las experiencias formativas en sentido amplio (educativos y de investigación, sin disociarlos). Y aquí la gran clave en el tiempo que dedicaba a la formación, a las discusiones, a la apuesta por procesos colectivos en los espacios educativos que tuvieran una mirada amplia de la realidad social por la que transcurren los/as niños, los/as docentes, las familias.

Queremos agradecer a las compañeras que nos aportaron sus palabras. Seguramente, muchas más personas hubieran querido y podido agregar mucho a este homenaje. 
Realmente no pudimos ponernos en contacto con todos/as porque los tiempos de entrega imprimieron su lógica en este trabajo. La intención es abrir una puerta, "invitar a jugar", sin olvidar aquello que fue conformando nuestras propias experiencias formativas, porque consideramos que en tiempos tan difíciles (en que las políticas académicas $-\mathrm{y}$ en lo que las políticas gubernamentales tratan de imponer-, cada vez tienen más lógica mercantilista e individualista, donde abundan los recortes presupuestarios para ciencia y técnica, para educación) es necesario tener presentes estas enseñanzas: estas apuestas por la producción colectiva, por el entrecruzamiento de campos, por la socialización de las producciones, pero también por rescatar y trasmitir la formación crítica y humana, cual proceso artesanal —siguiendo a Wright Mills (1979)—, que se va aprehendiendo en lo cotidiano, en el hacer, pensar, compartir y escribir; ejercicio incodificable para el que no hay una receta, sino múltiples aportes que llevan a la construcción colectiva. Esto último no como algo exclusivo de la lógica de trabajo de Liliana, sino como algo que comparten los equipos de la RIAE (a nuestro entender) y que por eso presenta como espacio tanta potencialidad.

La propuesta de Liliana Sinisi no empezaba ni acababa en el estudio de las problemáticas relacionadas con la "discapacidad" o con las "NEE"; nos invita a pensar críticamente la educación en sentido amplio, la conformación de los procesos escolares, de los sujetos que los atraviesan. En este sentido, consideramos que el mejor homenaje que podemos realizar es seguir la lucha contra los procesos de exclusión, discriminación, racismo, desigualdad, diferenciación; y seguir planteando una posición ética y de compromiso en los debates que en los actuales contextos sociohistóricos se encuentran signados por procesos sociopolíticos de profundización neoliberal y en los que se realizan fuertes críticas a la escuela pública, la cual para nosotras sigue siendo un espacio para la transformación social. 


\section{Q Bibliografía}

》 ACHILLI, Elena. 2005. Investigar en Antropología Social. Los desafíos de transmitir un oficio. Rosario: Laborde Editor.

" ACHILLI, Elena. 2010. Escuela, familia y desigualdad social. Una antropología en tiempos neoliberales. Rosario: Laborde Editor.

» EZPELETA, Justa y ROCKWELL, Elsie. 1985. "Educación y clases populares en América Latina”. En: M. Ibarrola y E. Rockwell (Comps.). Educación y clases populares en América Latina. México: DIE/IPN. pp. 14-42.

" MONTESINOS, Maria Paula y SINISI, Liliana. 2003. "Niñez, pobreza y diferenciación social”. Runa. Archivo para las ciencias del hombre, 24(1): 63-81.

" MONTESINOS, María Paula, SINISI, Liliana y SCHOO, Susana. 2009. Sentidos en torno a la "obligatoriedad" de la Educación Secundaria. Buenos Aires: DiNIECE, Ministerio de Educación de la Nación.

"NEUFELD, Maria Rosa y THISTED, Jens Ariel. 2004. “'Vino viejo en odres nuevos’: acerca de educabilidad y resiliencia”. Cuadernos de Antropología Social, 19: 83-100.

" ROCKWELL, Elsie. 1987. “Reflexiones sobre el proceso etnográfico, 1982-1985”. En: Documentos del DIE. №3. México. DIE/IPN.

》 ROMERO ACUÑA, Macarena. 2015. Políticas socioeducativas y trabajo docente en relación a la 'intregración-inclusión' de 'niños con necesidades educativas especiales'. Un análisis de procesos cotidianos en una escuela primaria. Tesis de Licenciatura, Universidad Nacional de Rosario.

" SINISI, Liliana. 1999. "La relación nosotros-otros en espacios escolares 'multiculturales'. Estigma, estereotipo y racialización”. En: M. R. Neufeld y J. Thisted (Comps.). “De eso no se habla..." los usos de la diversidad sociocultural en la escuela. Buenos Aires: Eudeba. pp. 189-234.

"SINISI, Liliana. 2010. "Integración o Inclusión escolar: ¿un cambio de paradigma?". Boletín de Antropología y Educación, 1: 11-14.

» SINISI, Liliana. 2012. "Políticas Socio-educativas: de la integración a la inclusión escolar. ¿Cambio de paradigma?”. Revista Espacios, 49: 59-70.

"SINISI, Liliana. 2013. “Contribuciones de la etnografía para el estudio de redes y tramas psico-educativas". En: N. Elichiry (Comp.). Historia y vida cotidiana en educación. Perspectivas interdisciplinarias. Buenos Aires: Manantial. pp. 49-66.

» WRIGHT MILLS, C. 1979 [1959]. La imaginación sociológica. México: Fondo de Cultura Económica. 
